\title{
ESPECIES NOVAS DE PSEUDOPHYLLINAE ESPECIALMENTE DO BRASIL (ORTHOPTERA, TETTIGONIIDAE) *
}

\author{
S. DE TOLEDO PIZA JR.**
}

Brachyauchenus varicosus sp.n.

o - Colore castaneo-picea. Caput parce sculpturatum Fas tigium verticis latum, concavum, apice modice diviso, margines scrobum antennarum superante. Frons nigro-nitida. Antennae pilosae, scapo valido, spina acutissima extus, tuberculo parvo intus armato. Pronotum dense rugulato-granulatum, margine antico in medio dente parvo, sulcis profundis, irregula ribus. Elytra extremitatem abdominis valde superantia, venis transversalibus precipue fortiter varicosis. Alae obscurae. Pedes rudini, dense pilosi. Femora antica compressa, basi modice arcuata, subtus spinulis 4-5 armata. Femora intermedia minus arcuata, spinis majoribus. Femora postica spinis 4-6. Tibiae intermediae supra inermes, tibiae posticae fortiter compressae spinis 10 magnis, triangularibus, acutis armatae. Lobi geniculares femorum spinosi, spina externa majore. Lamina supraanalis triangularis, apice obtuso, dimidium cercorum attingente. Cerci teretes, obtusi. Lamina subgenitalis parte distali angustata, subparallela, apice profunde U-incí so, stylis cylindricis, elongatis.

* Entregue para publicação em 28/08/80.

** Departamento de Zoologia, E.S.A. "Luiz de Queiroz, USP. 
$q$ - Mari similis sed major. Ovipositor modice curvus, acutus, superne prope apicem obsoletissime serrulatus, disco rugulis nonnullis. Lamina subgenitalis lata, triangularis vel foliacea, obtusa.

\begin{tabular}{|c|c|c|c|c|c|}
\hline & & o & & $q$ & \\
\hline Longitudo & corporis & 23 & $\mathrm{~mm}$ & 27 & $\mathrm{~mm}$ \\
\hline " & pronoti & 5 & & 6 & \\
\hline$"$ & elytrorum & 23 & & 27 & \\
\hline atitudo & 11 & 2,8 & & 2,5 & \\
\hline ongitudo & fem. ant. & 7 & & 8 & \\
\hline 11 & $"$ post. & 13 & & 15 & \\
\hline$"$ & ovipositoris & & & 10 & \\
\hline
\end{tabular}

Tipo: Um macho coletado no E. do Parā, por Silva Gomes, em 1977.

Alótipo: uma fêmea coletada juntamente com o macho.

Parátipo: uma fêmea de Mangabeira, E. de Alagoas, cole tada por A.C. Camargo, em 1952. Os tipos se encontram no Dep. Zool. da ESALQ, em Piracicaba, S.P.

Difere de Brachyauchenus castaneus Brunner, do qual se aproxima, pela fronte negro-brilhante, pelas varizes das veias elitrais e pelos espinhos dos lobos geniculares.

\section{Entacanthodes tesselalus sp.n. (Fig. 1)}

Castaneo-virescens, capite albescenti. Vertex convexus. Oculi rotundati, prominentes. Fastigium verticis triangulare, excavatum, vertice elevato, a fastigio frontis valde remoto, margines scrobi antennarum vix attingente, basi tuberculis 2 obscuris praeditum. Scapus antennarum intus ad api cem dente parvo, obtuso armatus, flagellum parte basali al= bicanti. Pronotum convexum, haud sculpturatum, non pellucidum, metazona vix elevata, leviter corrugata, margine antico rotundatim producto, margine postico rotundato limbato. Elytra cinereo virescentia, a medio sensim angustata, apice rotundata, ramo radiale sinuoso, in tertia parte apicali orien te, extremitatem abdominis valde superantia. Alae parte ant $\underline{\dot{i}}$ 
ca ocracea, disco invenuste tesselatae. Femora antica subtus spinis 3 , femora intermedia 4, femora postica 8, armata. Lobi geniculares-lobus externus femorum intermediorum exceptus - spinosi. Tibiae anticae supra inermes, intermediae spi na singula prope basim, posticae multispinosae. Cerci teres, crassi obtusi, lateraliter excavati. Lamina subgenitalis profunde U-incisa, ramis inferne sulcatis, stylis fortissimis, compressi, excavatis, apice acutissimis (Fig. 1).

$\begin{array}{clc}\text { Longitudo corporis } & 42 \mathrm{~mm} \\ \text { " } & \text { pronoti } & 10,5 \\ \text { " } & \text { elytrorum } & 48 \\ \text { Latitudo } & \text { " } & 8 \\ \text { Longitudo fem. ant. } & 13,5 \\ \text { " } & \text { " post. } & 28 \\ \text { " } & \text { stylorum } & 8\end{array}$

Pátria: Belèm, Parā.

Co1.: 1977.

Tipo: um macho no Dep. Zool., ESALQ, Piracicaba.

Difere das espécies conhecidas por ser muito maior, pelas asas grosseiramente tecidas de escuro e pela lamina subgenital dividida em dois longos lobos providos de estilos co mo na figura.

\section{Eumecopterus vetus sp.n.}

Gracillimus, stramineus, pubescens. Frons ampla, conve xiuscula, nitida, ocello rotundato-ovato, castaneo, antennis uniformibus, spina segmenti Ii magna, acuta. Fastigium verticis triangulare, elongatum, sulcatum, vertice acuto marginem scrobi antennarum superante. Fronotum fortiter granulatum, sulco postico profundo, metazona carinita. Elytra elongata, parallela, multo longiora quam latiora, apice modice obliquiter-rotundata, venis sat distinctis, longitudinalibus vix flexuosis, $s R$ valde post medium oriente, $M$ a $\mathrm{R}$ et $\mathrm{Cu}$ aequedistanti. Alae hyalinae, venis flavis. Pedes longes. Femora I spinis $5-7$, II spinis $1-2$, III spinis 10-11 armata sp 
nis omnibus dilutis, apice nigris. Femora postica dimidio ba sali faciei internae nigricantia necnon subtus ad faciem externam vitta basali castaneo-denso ornata. Lobi geniculares omnes spinosi. Tibiae intermediae superne inermes, posticae utrinque spinosae. Lamina supraanalis disco triangulari, sul cato, apice obtuso, marginibus perpendiculariter deflexis. Lamina subgenitalis lata, cordiformis, apice acuto, disco in medio obscuriore. Ovipositor longus, angustus, rectus, sine rugas preapicales.

$\begin{array}{clr}\text { Longitudo corporis } & 46 & \mathrm{~mm} \\ \text { " } & \text { pronoti } & 8,5 \\ \text { " } & \text { elytrorum } & 72 \\ \text { Latitudo } & \text { " } & 9 \\ \text { Longitudo } & \text { fem. ant. } & 18 \\ \text { " } & \text { " post. } & 32 \\ \text { " } & \text { ovipositoris } & 22 \\ \text { Latitudo } & \text { " } & 3\end{array}$

Pátria: Campos do Jordão, S.P.

Col. Spitz, XII - 1926

Tipo: uma fêmea, pertencente ao Museu de Zool. da USP, presentemente no Dep. Zool. da ESALQ, Piracicaba.

Parátipo: uma fêmea, de procedência desconhecida, no Museu de Zool. da USP.

A presente espécie é muito próxima de Eumecopterus nigrovittatus Brunner, diferindo por ser maior, por ter o pronoto fortemente granuloso, pelos élitros 9 vezes mais longos do que largos e principalmente pela lâmina supraanal de margens dobradas perpendicularmente para baixo e pela lâmina sub genital em forma de coração com o ápice pontudo.

GLophyraspis beieri sp.n. (Fig. 2)

Caput virescens, laeve. Vertex modicissime convexus, levissime puncturatus, fastigio cordiformi, sulcato, apice margines scrobium antennarum vix attingente. Frons lata, inae 
qualis, raro punctata, oculis mediocribus, non multo salientibus, antennis viridibus, gula nigerrima. Pronotum ocraceoviride, lobis deflexis longioribus quam altioribus, fortiter granulatis, margine inferiore lutescenti. Elytra viridia, Rs post medium oriente, ramis cum ramis transversis reliquarum venarum laxe reticulatis. Campus stridulatorius in elytro dex tro subopaco, colore castanea, in elytro sinistro speculo mag no, triangulato-rotundato. Alae hyalinae, venis $\mathrm{flavis,trans}$ versalibus sat dilutis. Femora virescentia, I et II spinis 4 concoloribus, apice nigris, in II majoribus, III spinis 8 nigris, recurvis, versus apicem crescentibus. Tibiae I super ne inermes, II spinis 5, III spinis complurimis. Lobi genicu lares, lobo interno femorum intermediarum exceptis, spinosis. Lamina supraanalis brevis, triangularis. Cerci cylindrici, crassi, apice obliquiter truncato, mucrone parvo intus armato. Lamina subgenitalis versus apicem angustata, apice subtruncato, stylis crassis, teretibus, nom multo elonga tis.

\begin{tabular}{|c|c|c|}
\hline Longitudo & corporis & 31 \\
\hline & pronoti & 8 \\
\hline " & elytrorum & 31 \\
\hline Latitudo & & 11,2 \\
\hline Longitudo & fem. ant. & 11 \\
\hline & stvli post. & $\begin{array}{r}21 \\
2\end{array}$ \\
\hline
\end{tabular}

Pátria: Serra do Caraça, Santa Bárbara, M.G.

Co1.: Araujo \& Martins, XI - 1960.

Tipo: um macho, do Museu Zool. USP, presentemente no Dep. Zool. da ESALQ, Piracicaba.

Distingue-se pela cor, pelas granulações do pronoto, pe los espinhos das patas, pela lâmina supraanel triangular e pela lâmina subgenital mais ou menos truncada e provida de estilos grossos e muito menores do que em G. laeta (Brunner). o nome da espécie é dado em homenagem ao distinto ortopterólogo vienense, Dr. Max Beier. 
Gongrocnemis belemita sp.n. (Fig. 3)

Castaneo vario. Vertex castaneo-surdus. Frons, clypeus, labrumque castaneo dentiore, nitida. Pars lateralis frontis lutea. Discus pronoti crebe granuloso-rugulosus, quam pars reliqua censior, et ab illis vittis luteis irregularibus parce distinctis sectis, margine antico rotundato, inerme, margine postico vix rotundato. Pronotum nitidum. Elytra nitida, maculis nudis obscuris nonnullis necnon reticulis albis inter venas partim ornata, margine postico castaneo-nigro interrupte ornato. Femora antica 3-4 spinosa, intermedia spinis 4, postica spinis 6-7, spinis omnibus concoloribus, apice obscurvoribus. Tibiae intermediae supra inermes. Pars ventralis abdominis longitudinaliter in medio castaneo-nigro-ni tida. Margo posticus segmenti abdominalis ultimi in mediova $\overline{1}$ de concavus. Lamina supraanalis triangularis, foveolata, api= ce angusto. Cerci crassi, apice incurvi, mucronati. Lamina subgenitalis convexa versus apicem modice angustata), U-incisa, stylis longis.

$\begin{array}{clc}\text { Longitudo } & \text { corporis } & 27 \mathrm{~mm} \\ \text { " } & \text { pronoti } & 6,5 \\ \text { " } & \text { elytrorum } & 29 \\ \text { " } & \text { fem. ant. } & 9 \\ \text { " } & \text { " post. } & 16,5\end{array}$

Pátria: Belém, E. do Pará.

Col.: Silva Garcia, XII - 77.

Tipo: um macho no Dep. Zool. ESALQ, Piracicaba.

Aproxima-se muito de G. azteca Sauss, et Pictet, da qual se distingue pela ausência de tubérculo no bordo anterior do pronotum e pela forma da lâmina supraanal.

Gongrocnemis montana sp.n. (Fig. 4)

Precedenti simillima differt pronoto disconitido, fortiter puncturato, margine antico prominenter angulato-rotundato, elytris dilutioribus, femoribus intermediis 4-spinosis, 
lamina subgenitalis profunde incisa, lobis acutissimis, disco ovipositoris in medio longitudinaliter luteo.

$\begin{array}{clc}\text { Longitudo } & \text { corporis } & 26 \\ \text { " } & \text { pronoti } & 5,8 \\ \text { " } & \text { elytrorum } & 22 \\ \text { " } & \text { fem. ant. } & 8 \\ \text { " } & \text { " post. } & 16,5 \\ \text { " } & \text { ovipositoris } & 10\end{array}$

Pátria: Serra do Navio, Amapá.

Co1.: Lenko, XI - 1957.

Tipo: 19 , pertencente ao Museu de Zool. da USP, no Dep. Zool. da ESALQ, Piracicaba.

Gongrocnemis surinama sp.n. (Fig. 5)

Colore castanea uniformi. Antennae concolores. Pronotum granulosum, granulis irregularibus humilibusque, margine antico rotundato, postico truncato. Elytra sine maculis. Alae cinereo-obscurae. Pedes inornati. Femora antica et intermedia 3-spinosa, femora postica spinis 7 concoloribus, apice castaneo densiore. Tibiae intermediae supra inermes. Lamina supraanalis triangularis, brevis, apice rotundato. Lamina subgenitalis convexa, brevis, U-incisa. Ovipositor subtus modice convexus, supra post medium concavus, dimidio apicali obscuriore.

$\begin{array}{clrl}\text { Longitudo } & \text { corporis } & 26 & \mathrm{~mm} \\ \text { " } & \text { pronoti } & 6 & \\ \text { " } & \text { elytrorum } & 20 \\ \text { " } & \text { fem. ant. } & 9 \\ \text { " } & \text { " poipositoris } & 18 & \end{array}$

Pátria: Suriname, Distrito Marowijne.

Co1.: B. Malkin, X - 1963.

Tipo: $1 \%$, pertencente ao Museu de Zool. da USP, no Dep. Zool. da ESALQ, Piracicaba. 
Parátipo: $1 \%$, bem menor, da mesma procedência, col. em VIII-1965, também pertencente ao Museu de Zool. da USP.

Aproxima-se mito de G. mutica Brunner, diferindo pelas antenas não anuladas, pelos lobos laterais do pronotonão escurecidos, pela lâmina subgenital curta, convexa, de bordos arredondados e com uma incisão em U e pelas menores dimensões dos élitros, das patas e do ovipositor.

\section{Jimenezia incognita sp.n. (Fig. 6).}

Statura mediocri, gracilis, colore laete viridi-tomentosa. Caput - partibus bucalibus ac segmentis antennarum I et II inclusis-albicans. Frons alta, lateraliter compressa. Flagellum antennarum utrinque castaneo-nigrum, scrobi antena rum marginibus supra tangentibus. Oculi magni, obscuri, paulo prominentes. Discus pronoti irregularis, indistincte punc turatus antice et postice rotundatus, sulco postico paulo post medium sito, lobis deflexis rotundatim insertis, angulo antico rotundato, postico recto. Elytra elongata, extremitatem femorum posticorum valde superantia, venis sat distinctis, $S c$ et $R$ parallelis, fere tangentibus, Rs valde post medium oriente, ultra medium diviso, ramo antico circa medium furcato. Femora antica et intermedia infra inermia, postica parce spinosa, spinis concoloribus. Tibiae intermediae supra spinulis paucis. Prosternum spinis binis, parvis, triangularibus. Segmentum abdominale dorsale ultimum postice angustatum, prominens, apice nigro-nitidum. Cerci filiformes, elongati. Ovipositor rectus, extremitatem femorum posticorum at tingens, valvis castaneo-marginatis, apice acuto, minutissi= me denticulato. Lamina subgenitalis brevis, apice obtuse rotundata.

$\begin{array}{clr}\text { Longitudo corporis } & 25 & \mathrm{~mm} \\ \text { " } & \text { pronoti } & 5 \\ \text { Latitudo } & \text { elytrorum } & 39 \\ \text { Longitudo fem. ant. } & 5 \\ \text { " } & \text { " post. } & 12 \\ \text { " } & 28 \\ \text { " tibiarum post. } & 30 \\ \text { Latitudo } & \text { ovipositoris } & 18 \\ \text { " } & 1,8\end{array}$


Pätria e coletor desconhecidos.

Tipo, uma fêmea, no Dep. Zool. ESALQ, Piracicaba.

Difere muito, por caracteres morfológicose pela coloração, da única espécie do gênero, descrita do Equador, Jimenezia elegans Bolivar (macho).

Paraemasia gen. n. (Platyphyllini)

Vertex convexiusculus, fastigio rhomboidali, excavata ba si tuberculis duobus remotis, apice acuto, margines scrobum antennarum attingente. Scapus antennarum dente apicali interno conico armatus. Frons lata, subplana, utrinque depressa. Pronotumbreve, rotundatum, tuberculis complurimis, humilibus, irre gularibus, margine antico curvatim prominenti, in medio denticú lato, utrinque vix sinuoso, margine postico metazonae truncató inermi, disco sulcis latis, sulco postico post medium sito, in lobis deflexis ampliato, lobis ipsis longioribus quam latioribus, marginibus inferioribus antice angulo recto, postice obtuso, orlatis, disco granulato et sulcato. Elytra lata, versus ex tremitatem regulariter attenuata, Sc et $R$ paralellis sed prope apicem disjunctis, $R$ ad extremitatem divisa, Rs valde post medium oriente, $\mathrm{M}$ a $\mathrm{R}$ quam a Cu remotiore, cum Rs subrectis.

Alae uniformes, hyalinae. Prosternumbispinosum mesoster num obsolete lobulatum, transversum, sulco lato ac profundo, praé ditum, metasternum latum, sulco profundo subrecto. Coxae norma les. Femora spinosa, spinis fortis. Lobi geniculares omnes spino si. Tibiae intermediae superne spinis raris, tibiae posticae sp $\bar{i}$ nis triangularibus, longis, acutis, remotis, internis majori= bus. Lamina subgenitalis lata, triangularis, apice obtuso. Lamina supraanalis brevis, basi convexa, prope apicem excavata, apice ipso bilobulato, lobis rotundatis. Ovipositor rectus, for tis, acutus, rugis discoidalibus praeapicalibus, margine supero post medium modice concavo et obsolete denticulato.

o presente gênero, difere do gênero Aemasia Brunner, pela cor castanha escura, pela forma do fastigio do vértice, que alcança as margens dos escrobos antenais, pelo escapo armado de pequeno dente, pelo pronoto provido de pequeno dente no meio do bordo anterior e desarmado no posterior, pelos éli tros com Rs: nascendo muito depois do meio, pelas coxas normais e todos os lobos geniculares armando de espinho.

Tipo: a espécie seguinte. 
Paraemasia paraibana sp.n.

$\varsubsetneqq$ - Characteribus generis, colore castaneo-vario, fron te rufescenti, nitida. Elytra dimidio basilari venis reticulisque ferrugineis deinde virescentibus, segmentis abdominalibus postice modice cinctis, segmento ultimo castaneo-nigro nitido, ovipositore castaneo-obscuro, dimidio apicali, nigro nitido. Femora antica inferne spinis parvis 5, intermedia spi nis marjoribus 4, postica spinis 6-8 remotis, curvis acutissimis. Tibiae intermediae spinis 2 superne ad basim marginis postici, tibiae posticae multispinosae.

\begin{tabular}{|c|c|c|}
\hline Longitudo & corporis & 38 \\
\hline " & pronoti & 8 \\
\hline$"$ & elytrorum & 34 \\
\hline Latitudo & elytrorum & 9 \\
\hline Longitudo & fem. ant. & 14 \\
\hline " & " post. & 25 \\
\hline " & ovipositoris & 14 \\
\hline
\end{tabular}

Pátria: Paraỉba, Brasil.

Co1.: Exp. Depart. Zoo1. VI - 1957.

Scopiorinus turrialbae sp.n. (Fig. 7)

$\delta$ - Mediocris, castaneo-virescens. Vertex convexiusculus, rudis sed sine granilis distinctis, linea longitudinali angustissima castaneo-nigra in medio ornatus. Frons subplana, lata, nitida, utrinque modice compressa, ad finem clypei depressa. Antennae ad articulationes segmentorum obscure anu latae, scapo dente apicali parvo armato. Fastigium verticis angusto, excavato, apice margines scrobum antennarum superan te. Pronotum rotundatum, dense granulato-corrugatum, sulcis transversis paulo profundis, sulco postico a impressione par va, castaneo-nigra, brevi, secto, metazona, quammesozona lon giore, margine postico curvo, margine antico regulariter procurvo, in medio tuberculato. Elytra extremitatem abdominis valde superantia, versus apicem modice angustata, apice ipso rotundato, $S c$ et $R$ usque ad quartam partem apicalem conjunctis, $R$ ramos 4 obliquiter emittente. Rami radiales a ramulis 
secundariis connecti cellulas formantes. Area costalis venis lo obliquiis, plusminusve parallelis praedita. Speculum sinistrum sub opacum, vix longius quam latius. Speculum dextrum nitidum, quam sinistrummajus, subaeque longusse et latum. Pedes graciles. Femora I et II spinis $4-5$ concoloribus, apice castaneo, femora III spinis 5-6. Lobus genicularis externus femorum anticorum et internus femorum II ac III spina armatus. Tibiae intermediae superne inermes. Lamina supraanalis lata, truncata. Lamina subgenitalis parte terminali angusta versus apicem nonnihil latiore, margine postico truncato utrinque lobo brevi rotundato praedito.

\% - Mari similis sed paulo major. Lamina subgenitalis transversa, margine postico in medio ample emarginato. Ovipo sitor valde arquatus, versus apicem fortiter attenuatus, ap $\bar{i}$ ce acuto, superne obsolete denticulatus, discis a parte dorsali basis versus partem ventralem extremitatis serie striarum transversalium praeditis, apicem elytrorum superans.

$\begin{array}{clrrrr} & & 0 & & & \\ \text { Longitudo } & \text { corporis } & 25 & \mathrm{~mm} & 27 & \mathrm{~mm} \\ \text { " } & \text { pronoti } & 4,5 & 5 & \\ \text { " } & \text { elytrorum } & 25 & 26 & \\ \text { Latitudo } & \text { " } & 6 & 7 & \\ \text { Longitudo fem. ant. } & 10,5 & 11 & \\ \text { " } & \text { " post. } & 20 & 21 & \\ \text { " } & \text { ovipositoris } & & 9\end{array}$

Pátria: Costa Rica, Turrialba.

Col.: Evôneo Berti Filho, 1937 (Março)

Tipos: um macho e uma fêmea, no Departamento de Zool. da ESALQ, Piracicaba, S.P., Brasil.

A presente espécie difere de Scopiorinus mucronatus (Sauss. \& Pictet) pelo espinho dos lobos geniculares e pela forma da lâmina subgenital do macho e da fêmea. 


\section{SUMMARY}

\section{NEW SPECIES OF PSEUDOPHYLLINAE SPECIALLY FROM BRAZIL (ORTHOPTERA, TETTIGONIIDAE)}

In this paper, the following new species of Pseudophyllinae (Orthoptera-Tettigoniidae) are drescribed, to one of which a new genus was erected: Brachyauchenus varicosus, Entacanthodes tesselatus, Eumecoptems vetus, Glaphyraspis beieri, Gongrocnemis belemita, Gongrocnemis montana, Gogroc nemis surinama, Gimenesia incognita, Paraemesia paraibana(n. g.), and Scopiorinus turrialbae. 

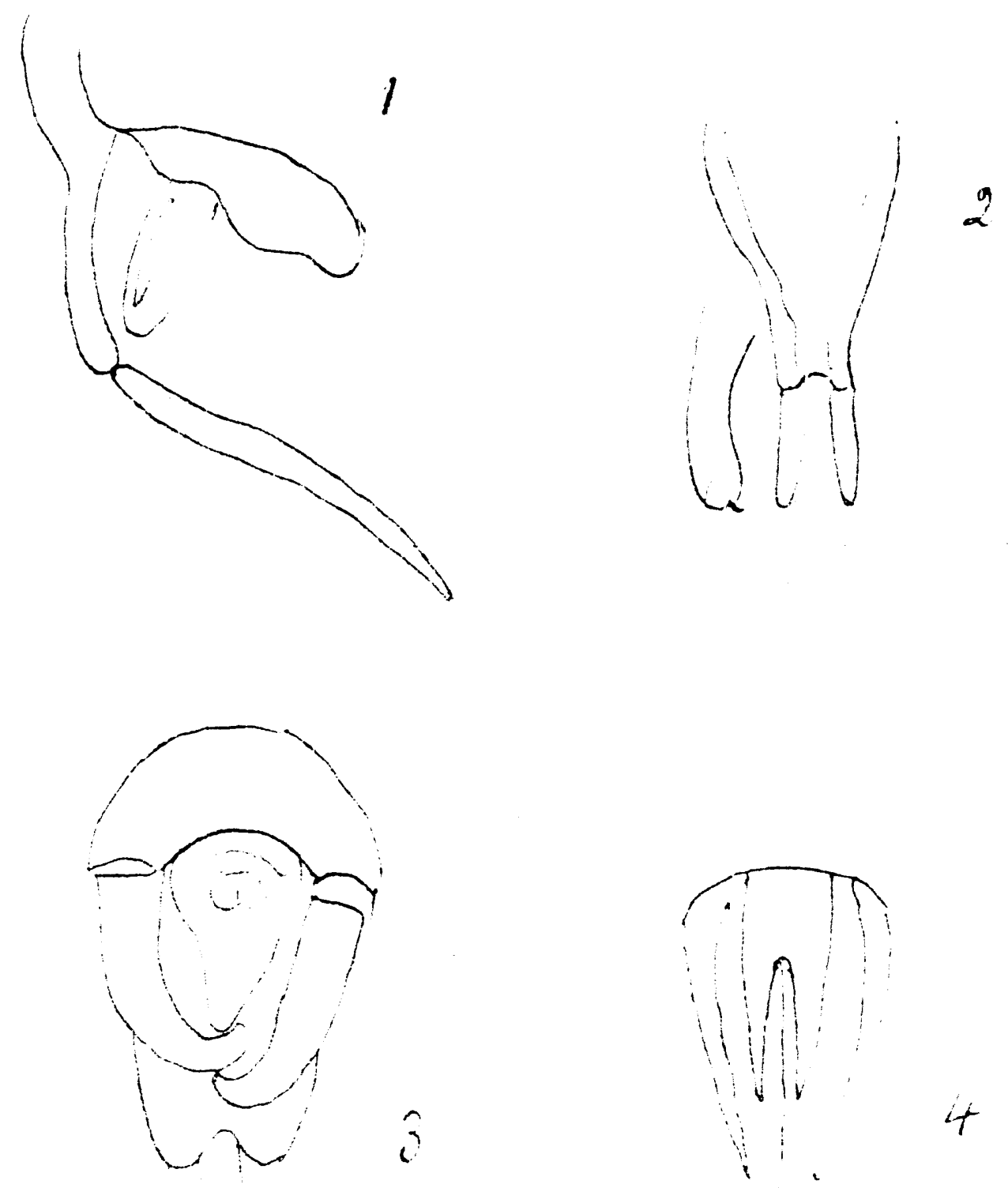

LEGENDA PARA AS FIGURAS: 1 - Entacanthodes tesselatus, macho: lobo da lâmina subgenital, estilo e cerco; 2 -'Glaphy rapis beieri, macho: cerco e lâmina subgenital; 3 - Gongroc hemis belemita, macho: lâmina supraanal, cercos e lâmina sub genital; 4 - Gongrocnemis montana, fêmea: lâmina subgenita $\overline{1}$ e cercos. 

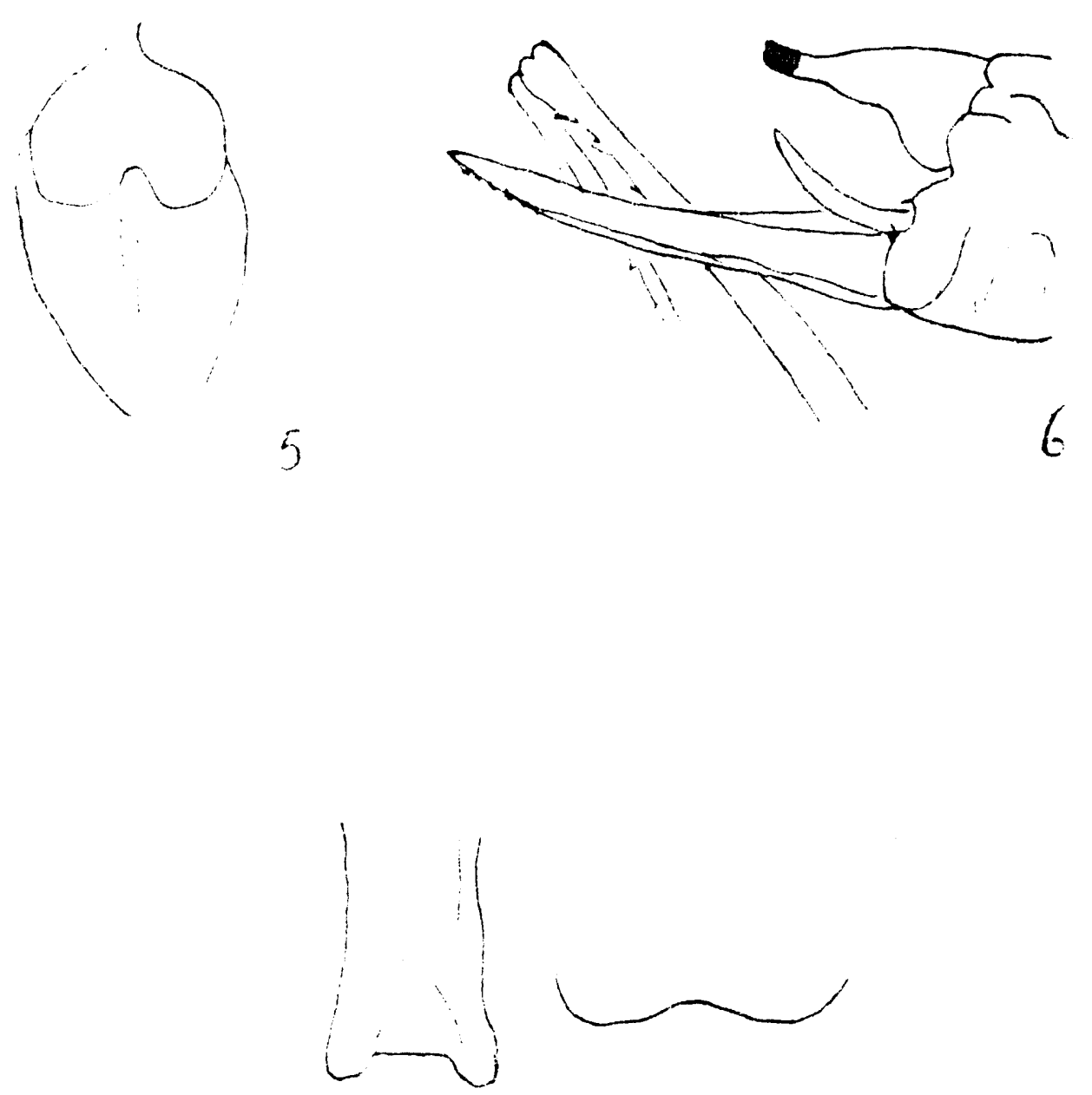

$y$

LEGENDA PARA AS FIGURAS: 5 - Gongrocnemis surinama, fêmea: lâmina subgenital e base do ovipositor; 6 - Jimenesia incognita, fêmea: lâmina subgenital, cerco, ovipositor e base de uma pata traseira; 7 - Scorpiorinus turrialbae, macho e fêmea: lâmina subgenital. 